\title{
Movimientos migratorios en el s. XXI
}

\section{Migratory movements in the s. XXI}

\author{
Florinda Rojas \\ Universidad de Georgetown, Estados Unidos de América \\ florrojas@hotmail.com $\backslash$ https://orcid.org/0000-0002-5376-160X
}

Fecha de recepción: 29 de marzo de 2021

Fecha de aceptación: 6 de abril de 2021

Fecha de publicación: 1 de julio de 2021

Favor citar este artículo de la siguiente forma:

Rojas, F. (2021). Movimientos migratorios en el s. XXI.

AULA Revista de Humanidades y Ciencias Sociales, 67 (2), 79-88

https://doi.org/10.33413/aulahcs.2021.67i2.182

\section{RESUMEN}

El estado y características de las migraciones en un mundo cada vez más influenciado por los efectos globalizantes, nos hace reflexionar sobre las oportunidades y desafíos que los movimientos migratorios nos plantean. Cada vez, se observa un incremento en el número de migrantes, con modalidades y modo operacional, que se intensifica con el apoyo de diferentes redes sociales. Esta situación viene a extremarse, debido a la crisis sanitaria mundial, que representa el virus del COVID-19, como también, debido a las nuevas corrientes migratorias vinculadas con los desplazamientos humanos por razones socioeconómicas, graves violaciones a los derechos humanos y también, como resultado de los efectos del cambio climático.

Palabras clave: Comunidad, derechos humanos, desarrollo, movimientos, migración.

\begin{abstract}
The state and characteristics of Migration, in a World increasingly influenced by globalizing effects, makes us reflect on the opportunities and challenges that migratory movements pose to us. Each time, there is an increase in the number of migrants, with modalities and operational mode, which intensifies, with the support of different social networks. This situation is exacerbated, due to the global health crisis, represented by the COVID -19 virus, as well as due to the new migratory flows linked to human displacement for socio-economic reasons, serious human rights violations and also, as result of the effects of climate change.
\end{abstract}

Keywords: Community, human rights, development, movements, migration. 


\section{Introducción}

Los movimientos migratorios que se registran a inicios del siglo XXI, son el efecto de largo plazo de acontecimientos geopolíticos, bélicos y climáticos de gran impacto regional y global que se han sucedido desde finales del siglo anterior hasta el día de hoy. Esta afirmación se basa en hechos tales como el aumento en el tráfico de personas, desde África hacia Europa, vía marítima; el mayor desplazamiento humano jamás visto en América Latina, desde Venezuela; la movilización de personas de diferentes naciones, desde Centroamérica, vía terrestre, con destino a México y los Estados Unidos de América; así como la migración forzada y de refugiados, principalmente desde Siria, víctima de la guerra híbrida que las grandes potencias libran en su territorio desde hace casi 10 años. Pero no menos importante es el desplazamiento forzado, tanto interno como externo, que se está produciendo como consecuencia del cambio climático y las catástrofes naturales súbitas provocadas por el calentamiento global, que activan movimientos migratorios imprevisibles con el potencial de transformar completamente los perfiles demográficos de regiones enteras.

A su vez, en el Caribe Insular continúa el éxodo de personas desde Cuba, vía marítima, de manera clandestina. Obviamente, las salidas de personas desde Cuba están vinculadas a persecución política, por lo que cada caso, tiene el mérito de ser analizado propiamente, a la luz de lo que establece la Convención sobre Refugiados del 1951. En relación a la emigración desde República Dominicana, si bien la mayoría de las personas son sus nacionales, también se han identificado personas de nacionalidades diversas, lo que indica que el país continúa sirviendo de puente para la migración irregular. Por otro lado, el deterioro social y político generado por la pandemia en Haití, ha agravado la complejidad del flujo migratorio intrainsular.

Como parte de los programas y proyectos puestos en marcha por la cooperación internacional al desarrollo, las instituciones responsables de la gestión migratoria de los diferentes países han recibido asistencia técnica de expertos y asesores que han contribuido en la construcción de respuestas humanitarias a las crisis de movilidad humana. Asimismo, a la redefinición de nuevos enfoques respecto a los paradigmas de gobernanza migratoria y, en particular, a los aportes de la mano de obra extranjera y su relación con el mercado de trabajo de la comunidad receptora, de tal suerte que se entienda que esencialmente, se trata de una dinámica "ganar-ganar."

Si bien es cierto que los gobiernos y los organismos internacionales, despliegan ingentes esfuerzos por encontrar soluciones conjuntas a los desafíos que plantea la inevitable realidad de las migraciones, la intensidad del flujo solo ha podido ser ralentizada por la pandemia, pero desde el segundo semestre de 2020 ya se vienen presentado señales de reactivación.

\section{El drama humano de las migraciones en el contexto internacional}

Mientras iniciaba la redacción de este artículo, encontré dentro de mis apuntes una publicación del diario El País, de España, cuyo contenido era más o menos así:

"Hace poco nació Yambambi Yete, una bebé, en un barco italiano. Su madre, acababa de ser rescatada de una lancha llena de inmigrantes africanos, ésta era una más de las embarcaciones lanzadas al mar durante los últimos meses con destino a Europa. Los marinos, junto al capitán del barco de rescate, le dieron la bienvenida a la bebé con canciones italianas".

Esta historia que parece con final feliz no es siempre así, es únicamente una nota emotiva que tuvo lugar durante momentos que se revelan muy graves para la humanidad; se trata de lo que viene ocurriendo recurrentemente en el mar Mediterráneo, en el que miles de hombres, mujeres, niños y niñas, se aventuran en frágiles embarcaciones desde la costa africana (Libia, Marruecos, et.), atravesando el Mediterráneo hasta llegar a Europa.

Si bien no es la primera vez que se presentan estas oleadas de inmigrantes con des- 
tino a países europeos; es cada vez más significativo el número de personas que deciden, a todo riesgo, negociar con los traficantes de personas o con sus intermediarios, en la búsqueda de seguridad y una vida mejor; es la clásica lucha por la sobrevivencia. Pero también los conflictos armados de larga data, la desestabilización política y las guerras, continúan provocando el éxodo de personas en todas las latitudes del mundo. Lo que sumado a las nuevas corrientes migratorias como consecuencia de los estragos del cambio climático, constituyen tragedias migratorias, o modalidades impensadas como consecuencias de los cambios repentinos y cada vez más frecuentes en el medio-ambiente.

En el caso de Europa, la modalidad del reparto de personas inmigrantes entre estos países asociados bajo la bandera de la Unión Europea, y el consabido esfuerzo por contar con los operativos de rescate en alta mar, la vigilancia de las fronteras tanto marítimas como terrestres y la incorporación de un presupuesto permanente en los fondos estatales, no se han hecho esperar.

En América, los migrantes han venido trasladándose en dirección sur-norte, en busca del sueño dorado norteamericano o el estado de bienestar europeo; pero también se mantienen importantes flujos intrarregionales en el Caribe, el llamado Triángulo Norte y América del Sur.

Ciertamente, estas tendencias se continuarán profundizando si se toman en cuenta los impactos sociales que en los países en desarrollo provocará el estancamiento económico proyectado para el resto de la década. Veremos flujos más o menos elevados, dependiendo de la evolución de los factores antes mencionados, pero también, del éxito de los controles migratorios y de cuán efectiva pueda ser la lucha contra las redes de tráfico ilícito de personas.

No hay soluciones fáciles. Siempre será mejor iniciar la ayuda con una respuesta solidaria y humanitaria, que adoptar enfoques que revictimizan. No podemos ser indiferentes al dolor y la muerte que acecha a los náufragos que intentan escapar de la inanición en
África o a las familias que huyen de la violencia en Centroamérica.

Es que mientras la comunidad internacional no reconozca que, para que cada ciudadano proveniente de los países en desarrollo permanezca en sus lugares de origen, no habrá solución intermedia posible si no se atienden las raíces del problema. Y la única forma, consiste en apoyar programas de pacificación, enlazadas con apoyo económico para el desarrollo productivo de estas naciones pobres.

\section{Las migraciones en América Latina y el Caribe}

Los informes de las agencias especializadas de las Naciones Unidas (OIM, ACNUR) plantean la realidad de las migraciones de la siguiente manera:

"El panorama migratorio en América Latina y el Caribe ha cambiado rápidamente en la última década. Entre 2010 y 2019, el número de inmigrantes en la región aumentó en un $66 \%$, mientras que el número de emigrantes aumentó un 26\%. Unos 42,7 millones de personas viven fuera de sus países de nacimiento. Esto se debe principalmente a la migración venezolana, la cual constituye un reto que mantendrá en jaque a todos los países de la región por largo tiempo."

A fines de 2019, había un estimado de 4,8 millones de migrantes venezolanos, incluyendo refugiados y solicitantes de asilo. Aproximadamente el $85 \%$ de los que huyeron por factores de tipo económico, social o político en Venezuela, se han trasladado a otros países de la región. Colombia, país que comparte más de 2.000 kilómetros de frontera con Venezuela, ha recibido más de 1,6 millones de venezolanos desplazados por la crisis humanitaria en ese país.

América Latina y el Caribe, a su vez, han sido testigos de otros desafíos, pues en el llamado Triángulo del Norte de América Central en años recientes, se han intensificado los movimientos migratorios desde Honduras, El Salvador, Nicaragua y Guatemala. La mayoría de estos migrantes, son personas en condiciones de vulnerabilidad como mujeres, 
personas mayores, niños y adolescentes no acompañados.

Estos movimientos aparentemente espontáneos, cuya raíz deviene de la pobreza, desempleo y necesidades básicas no resueltas, han traído como consecuencia tensiones en las relaciones entre los países expulsores de personas -como los mencionados anteriormente-, así como con los países receptores, Estados Unidos de América y México, principalmente.

Si bien se afirma que los migrantes contribuyen al desarrollo, los países de destino tienden a asegurar que traen una enorme carga, hasta tanto tengan éstos la capacidad de devolver con sus contribuciones laborales lo que se ha invertido en ellos.

Pero aprovechar el potencial de desarrollo de la migración, se requiere de un vasto programa de inclusión, entendiéndose esto como la responsabilidad que deben tener los Estados receptores, para permitir la integración socioeconómica y la cohesión social de los migrantes.

Un plan de capacitación técnica y educación, por ejemplo, podría facilitar que los migrantes se inserten en el campo laboral. En un momento en que el potencial impacto adverso de la inmigración es una preocupación para muchos países, -afirma un informe de la ONU- debemos asegurarnos de que la migración respalde el desarrollo.

Estas políticas de integración socioeconómica también deben venir con medidas específicas para promover la convivencia pacífica entre los nativos del país y los inmigrantes, garantizando que los países de acogida se responsabilicen de los derechos de los migrantes, activando mecanismos en los medios sociales, así como en los círculos académicos, destinados a combatir todas las formas de discriminación y trabajo forzoso.

En este sentido, hay que reconocer, que varios países de la región han llevado a cabo campañas de sensibilización pública explicando los motivos que tuvieron esas personas para abandonar sus países y cómo contribuyen positivamente a las comunidades de acogida; aunque esto debe ser de modo permanente, de manera que, a mediano y largo plazo, las comunidades respeten y apoyen a los migrantes al reconocerlos como sus iguales.

Por otro lado, para evitar el resentimiento y la xenofobia, las poblaciones nativas necesitan ver los beneficios directos de la inmigración. Esto implica que los programas diseñados para apoyar a los migrantes resulten elaborados de manera mixta, es decir, que incluyan a las comunidades de acogida vulnerables y que los programas de inversión contribuyan al desarrollo de servicios públicos e infraestructura para lidiar con el rápido aumento de la población.

\section{Desafíos regionales}

La Cumbre del Foro Mundial sobre Migración y Desarrollo, celebrada en Quito el pasado mes de enero, ha sensibilizado a la comunidad internacional sobre los desafíos de migración y desarrollo en la región. Reconoció que, además de la ayuda humanitaria, la migración es un asunto de desarrollo y que los países deben invertir en programas de generación de ingresos para permitir a los migrantes, incluidos los refugiados y solicitantes de asilo, integrarse en los mercados laborales de sus países de acogida.

También requieren inversiones masivas en infraestructura y desarrollo local para ayudar a crear empleos que puedan absorber tanto a las poblaciones migrantes como a las de acogida. Pero, debido a que esto implica recursos financieros que la mayoría de los países de la región no tienen, la cooperación internacional necesita aumentar su cartera de inversión para ayudar a convertir la migración en una oportunidad para el desarrollo.

La migración está en aumento en la región y, para los países que optan por acoger a los migrantes de manera productiva e inclusiva, representa oportunidades de desarrollo y gobernanza. El PNUD, con su Centro Regional para América Latina y el Caribe y sus 26 oficinas en los países, está listo para apoyar a las autoridades nacionales y locales. 


\section{Informe de la CEPAL}

El panorama migratorio en las últimas décadas ha cambiado de características, es por ello que diversos investigadores y estudiosos sobre la materia hanpublicado documentos dirigidos a los tomadores de decisión para contribuir a que puedan hacer frente a estos nuevos desafíos; pero también, para que rescaten los beneficios que una migraciónorganizadaydocumentadapuedaaportaral desarrollo de los países de la región. Asimismo, se hace necesario promover $\mathrm{y}$ fortalecer el diálogo y la cooperación multilateral, desde la óptica de los derechos humanos de los migrantes y susfamilias, como forma legítima de lograr quelamigración internacional contribuya aldesarrollo de los países de América Latina y el Caribe.

Los distintos factores económicos, sociales, políticos y culturales vinculados a los movimientos migratorios, ponen de relieve la variedad de significados de este fenómeno. Por ende, su tratamiento impone profundos desafíos, por lo que es necesario avanzar en la formulación de programas de gobernabilidad para lograr una migración ordenada, segura y protectora de los derechos de las personas.

Lo primero que cabe mencionar en este plano es el papel que desempeñan las brechas de ingreso y los ciclos macroeconómicos desde el punto de vista de la doble causalidad entre migración y desarrollo, que se ha reflejado en la magnitud y dirección de las corrientes migratorias (Solimano y Watts, 2005). Específicamente, la demanda creciente de mano de obra para fomentar la competitividad e impulsar el crecimiento de la economía, por una parte, y la oferta de trabajo proveniente de las economías menos desarrolladas o que se encuentran en condiciones de mayor pobreza, por la otra, parecieran ser dos partes de una ecuación que debería resolverse sin mayor conflicto. Sin embargo, lasprogresivasrestriccionesalingresode inmigrantes (Naciones Unidas, 2004), la discriminación en las sociedades de llegada y las dificultades que enfrentan para ejercer sus derechos, son elementos que obstaculizan el desarrollo de esta relación en condiciones de mayor fluidez.

Un segundo tema relevante, que se ha analizado tradicionalmente es que no todos los inmigrantes convergen en la base de la pirámide ocupacional: existe un número importante de trabajadores altamente calificados que son empleados por grandes compañías, empresas e industrias. Los beneficios que ello acarrea para ambas partes son ampliamente reconocidos. La interpretación negativa de los altos costos sociales y económicos que significa la fuga de cerebros (brain drain) para los países emisores ha dado paso a una visión más positiva, según la cual los inmigrantes pueden convertirse en una especie de puente para el traspaso de tecnología desde el mundo desarrollado hacia las economías menos desarrolladas (Pellegrino, 2000). Sin embargo, subsisten evidencias de las pérdidas que supone el éxodo de intelectuales y existe preocupación ante el reclutamiento de personas calificadas de países del tercer mundo por parte de las grandes empresas del mundo desarrollado.

Un tercer elemento de interés en el plano económico, y de creciente importancia para la economía de algunos países emisores de migrantes, es el envío de remesas. Estas no deben analizarse solo desde una óptica económica, ya que influyen en múltiples aspectos tanto sociales como culturales e incluso políticos, y aún están en discusión sus posibles efectos en el desarrollo de los países que las perciben. Sin embargo, dado que estos envíos de dinero afectan la manutención o el incremento del nivel de consumo de las familias receptoras, han surgido dudas respecto de sus consecuencias en otras esferas sociales y familiares: ¿Es posible que la recepción de estos ingresos sea positiva para el desarrollo del país?; desde el punto de vista de los hijos y la familia, ¿cuáles son los costos sociales del hecho de tener madres o padres que deban emigrar hacia otros países para satisfacer las necesidades mínimas del grupo familiar? Estos son solo algunos de los problemas relacionados con el envío de reme- 
sas y que obligan a examinar detenidamente esta materia y sus múltiples repercusiones.

La mano de obra extranjera ha sido fundamental para el crecimiento económico; aporte que generalmente no se ha visto refrendado con un mayor reconocimiento de los derechos y la protección de los trabajadores migrantes y sus familias.

La relación entre migración y globalización no se restringe al acontecer de las últimas décadas del siglo $\mathrm{XX}$, y a las primeras del siglo XXI, sino que ha formado parte del proceso de modernización y ha desempeñado un papel central en la expansión y el desarrollo del capitalismo moderno, siendo más notoria en la movilización y provisión constante de mano de obra barata y especializada. Esta fuerza de trabajo se fue absorbiendo en las distintas instancias de poder económico y político: desde el sistema esclavista del Nuevo Mundo hasta la migración indocumentada, pasando por los programas de trabajadores invitados que se aplicaron en casi todos los países industrializados -Alemania, Bélgica, Inglaterra, Suiza y Francia, entre otros-, el Programa Bracero (Mexican Farm Labor Program) para los mexicanos en Estados Unidos y los actuales programas de selectividad migratoria en los que se recluta a los mejores estudiantes y recursos humanos calificados.

\section{Migración y gobernabilidad}

La agenda política en los años 90, las grandes transformaciones que provoca la migración internacional en la economía, la sociedad y la cultura de los países de origen, tránsito y destino se reflejaron con mayor fuerza y reiteración en los programas políticos gubernamentales, regionales, de la sociedad civil y de los organismos internacionales. La incorporación progresiva de estos temas condujo a que se prestara atención a numerosos aspectos relacionados con la migración, considerando su orientación desde arriba, así como las iniciativas desde abajo.

Los países de América Latina y el Caribe no están ajenos a las transformaciones e iniciativas en favor de la gobernabilidad compartida de la migración internacional.
Muchos participan activamente en foros intergubernamentales sobre la materia y han trazado una trayectoria que incluye logros, avances y desafíos. Al cabo de varios años, se observa que las distintas experiencias encaminadas a impulsar la gobernabilidad de la migración a escala regional o temática, se han emprendido siguiendo algunos lineamientos de carácter multilateral y global no exentos de cuestionamiento. Si bien los organismos internacionales han defendido estos principios propios de las Naciones Unidas, su trabajo concreto se ha visto muy afectado por la superposición de actividades entre sí, lo cual -como lo destaca el informe de la Comisión Mundial sobre las Migraciones Internacionales (GCIM, 2005) - indica la necesidad de buscar mecanismos sustantivos de coordinación y complementación.

En la actualidad, como lo señaló Lelio Mármora, para lograr la gobernabilidad migratoria se requiere legitimidad y eficacia, dos condiciones inherentes tanto a la democracia de cada sociedad como a la gestión migratoria nacional. La legitimidad exige, por ejemplo, que exista transparencia en el manejo de las políticas migratorias, y esta será mayor en la medida en que se promueva la participación de la sociedad civil en la toma de decisiones sobre la materia. Las violaciones de los derechos humanos de las personas son una muestra flagrante de la falta de transparencia (Mármora, 2002).

En la región, uno de los problemas que dificulta la coordinación entre los Estados, es que estos carecen de una política migratoria asertiva. Los asuntos migratorios dependen de una serie de instituciones y Sectores como Interior, Cancillería, Educación, Salud y Policía, entre otros-, que aportan visiones parciales y lógicas reactivas.

Otro de los inconvenientes es que los gobiernos locales — sobre todo los municipales-deben convivir y resolver directamente las situaciones problemáticas derivadas de la migración, pero no participan en la elaboración de los programas y políticas nacionales. Además, en la formulación de las políticas pertinentes los gobiernos no siempre inclu- 
yen a los actores importantes de la sociedad civil, tales como sindicatos, empleadores y asociaciones de migrantes.

En mayo de 2000, en Buenos Aires, se constituyó oficialmente la Conferencia Sudamericana sobre Migraciones (CSM), definida como un foro de coordinación y consulta integral en materia migratoria, cuyo objetivo es aunar esfuerzos para garantizar la protección, defensa y promoción de los derechos de los migrantes e intensificar la cooperación regional. Los analistas coinciden en señalar que este proceso consultivo se presenta en un marco de igualdad de poder entre los Estados, lo que contribuiría a fomentar el tratamiento mancomunado de algunos temas (Gurrieri, 2005). En la Declaración final de esa reunión se destacó que "la protección de los derechos humanos sigue constituyendo una preocupación fundamental para los paises de la región. En este sentido, los programas de difusión de los derechos de los migrantes a través de los Gobiernos y la sociedad civil, la concertación entre países receptores y emisores de migrantes, la promoción de migración ordenada y planificada como solución a la migración irregular y la lucha contra el tráfico de seres humanos, emergen como políticas responsables" (Buenos Aires, 2000). A su vez, los delegados de los gobiernos declararon que el adecuado tratamiento de la temática migratoria demanda un gran esfuerzo de modernización de su gestión, el fortalecimiento institucional de los organismos nacionales con competencia en estos temas, la capacitación de los funcionarios públicos, la actualización de las legislaciones nacionales y el desarrollo de mecanismos de coordinación entre los Estados participantes (www.osumi-oim.org).

Nuevas corrientes migratorias en la región De acuerdo con la CEPAL, el número de migrantes de Latinoamérica y el Caribe alcanzó un total estimado de 21 millones de personas en el 2000 y estimaciones hablan de casi 28 millones en 2005, éstos representan el $13 \%$ de los migrantes en el mundo y el $4,1 \%$ de la población en la región. De los 25 millones,
9 millones corresponden a México, seguido por los países de la Comunidad del Caribe, Colombia y algunos países andinos como Bolivia y Ecuador que exceden el millón y medio de personas. De acuerdo con Canales (2006) uno de cada dos migrantes en Estados Unidos proviene de América Latina.

La emigración desde América Latina coincide con el crecimiento exponencial de la inmigración en Europa y Estados Unidos desde otros continentes. La Europa unitaria nace precisamente cuando los flujos de inmigrantes de otros continentes se hacen cada vez más presentes y cuando empieza a sonar cada vez más en el discurso político la necesidad de pensarse como naciones multiculturales ${ }^{1}$.

En las últimas dos décadas las remesas enviadas hacia América Latina se han incrementado diez veces. Paralelamente, ha crecido el interés por el impacto de estos flujos en el desarrollo económico de los países receptores.

Según el Banco Mundial (2007) y el BID (2007), 5 la región encabeza el ranking mundial de receptores de remesas. No obstante, los efectos de las remesas varían de acuerdo con la magnitud de estas y al tamaño de la economía nacional. Por ejemplo, en el 2004 las remesas representaron el 16\% del Producto Interno Bruto (PIB) de Honduras y el 15\% de El Salvador. En los países más grandes, que son lo que perciben un mayor volumen de remesas, estas representan menos del 5\% del PIB nacional. En el caso de México, suponen solo el 1,6\% del PIB, mientras en Brasil corresponden al $0,4 \%$ del $\mathrm{PIB}^{2}$.

A pesar de su importante crecimiento, su impacto en la inversión y el crecimiento económico ha sido relativamente pequeño. Se trata más bien de transferencias directas a nivel micro, en el marco de las estrategias económicas familiares. En efecto, la mayor parte de las remesas que llegan a América Latina son utilizadas en gasto corriente o diario tanto en los casos de migración antigua, como la mexicana, o de migración reciente, como la ecuatoriana. 
Iniciativas mundiales $\mathbf{y}$ regionales sobre las migraciones: El Foro Mundial sobre Migración y Desarrollo

El Foro Mundial sobre Migración y Desarrollo es una iniciativa de Naciones Unidas. Aunque los gobiernos son los protagonistas que inciden en este espacio, las decisiones adoptadas en el seno del mismo no son vinculantes para los Estados. Es más bien un mecanismo de diálogo que permite analizar los desafíos migratorios y formular estrategias de gestión, desde un enfoque integrado que tome en cuenta el carácter multidimensional de las migraciones.

Compartir experiencias desde el Foro, ha posibilitado un entendimiento más objetivo de la realidad de los movimientos migratorios, así como la identificación de áreas de cooperación a nivel bilateral y regional.

Obviamente, el acompañamiento de la ONU a este proceso ha permitido su continuidad. La discreta participación de la sociedad civil, de los sectores empresariales y de organizaciones sindicales, ha permitido el análisis desde una perspectiva holística, en la que todos los sectores de la economía, la política y el desarrollo se conjugan desde diferentes ópticas. También ha sido clave la participación de observadores y expertos sobre temas de igualdad de género, economía, desarrollo, trabajo, relaciones exteriores, entro otros; lo que le ha permitido a este cónclave elaborar recomendaciones destinadas a los gobiernos con la finalidad de fortalecer la gobernanza migratoria.

\section{Conclusiones}

Elaborar estrategias para un plan de diseño migratorio depende de muchos factores, uno de ellos consiste en despolitizar el tema y dejarlo bajo la absoluta responsabilidad de los Estados. Al mismo tiempo, facilitar el acceso en la elaboración y puesta en marcha de programas y proyectos de apoyo, en la que participen activamente las instituciones de la sociedad civil especializadas en la materia. En ese sentido, debe existir el interés primario de colocar a las personas por encima de las diferencias políticas. En particular, asumiendo la defensa y protección de los derechos humanos de los migrantes y sus familiares.

La creación de un régimen de bienestar social debe ser inclusivo, integrador, y eficiente en asegurar la protección de las personas, especialmente de aquellas cuyas condiciones de vulnerabilidad sean más extremas. Si bien es cierto que los migrantes salen de sus países en búsqueda de mejores condiciones de vida, por ausencia de trabajo, servicios sociales básicos, entre otros, es también cierto que esa mano de obra puede ser capitalizada en función de las necesidades de los países cuyas economías son más abundantes que la de los países de origen. Desde esa perspectiva, la capitalización de los inmigrantes debe empezar por la regularización de su estatuto jurídico, y a seguidas, la incorporación de planes y programas de educación y formación técnica, para que éstos se incorporen en el tren de la economía de esos países receptores, ya que en la mayoría de ellos, esa mano de obra es esencial, no en detrimento de los locales, sino en respaldo a los nacionales. Es decir, es la suma de esfuerzos conjuntos en ruta al progreso de los países. 


\section{Referencias}

Canales, A. (2006). Panorama actual de las migraciones en América Latina. En G. Delgado (Eds.), Reseñas (pp.193-199). Universidad de Guadalajara.

Comisión Mundial sobre las Migraciones Internacionales. (2005). Las migraciones en un mundo interdependiente. https:// www.iom.int/es/comision-mundial-sobre-las-migraciones-internacionales-cm$\underline{\text { mi }}$

Gurrieri, J. (2005) Los procesos migratorios actuales en Sudamérica [Ponencia]. III Reunión de la Comisión de Laborales, Previsión Social y Asuntos Jurídicos del Parlamento Latinoamericano, Buenos Aires. http://www.conapo. gob.mx/work/models/CONAPO/mi- gracion internacional/migint desarro$\underline{110 / 14 . p}$

Mármora, L. (2002).Las políticas de migraciones internacionales. Paidós

Organización de las Naciones Unidas (2000). Conferencia suramericana sobre migraciones www.osumi-oim.org

Pellegrino, A. (2000). La migración internacional en América Latina y el Caribe:t tendecias y perfiles de los migrantes. Serie Población y Desarrollo. CEPAL

Solimano, A., \& Watts, N. (2005)International Migration, capital Flows and the global economy:lom run view. Serie Macroeconomía del desarrollo CEPAL, 35

\section{Notas}

${ }^{1}$ Ver Herrera Gioconda e Isabel Yépez, (2007) "Introducción". Nuevas migraciones latinoamericanas a Europa. Balances y desafíos. Quito: FLACSO Ecuador - UCL UB. OBREAL.
${ }^{2}$ Banco Mundial (2007) Cerca de casa: el impacto de las remesas en América Latina. Washington, DC. Banco Mundial y BID-FOMIN (2007) Remesas 2006. Remesas y Desarrollo, Washington, DC, marzo.

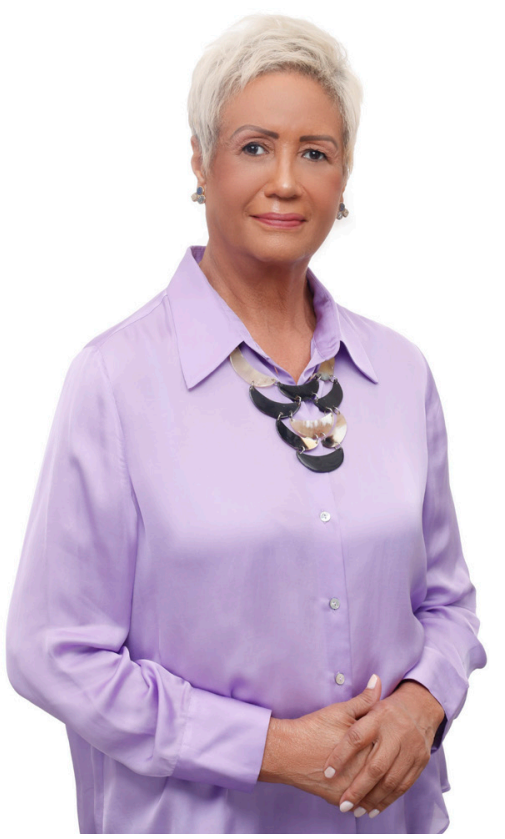

\section{Florinda Rojas}

Doctora en Derecho en la Universidad Autónoma de Santo Domingo (UASD). Realizó cursos de especialización en el Instituto Interamericano de Derechos Humanos en Costa Rica, la Universidad de Wisconsin y la Universidad de Georgetown en EEUU. Representante del Alto Comisionado de Naciones Unidas para los Refugiados (ACNUR) en varios países de América Latina y África. Tiene experiencia en el diseño de estrategias de negociación para la firma de la Ley de Refugiados en países del centro y el sur de América. Creó el Foro Regional para las Mujeres Refugiadas (FOREFEM). Desarrolló el Programa de Capacitación sobre los Derechos de las Mujeres para las Refugiadas en Zambia, África (1993-1995), y participó en la redacción de la iniciativa jurídica para la incorporación de los aspectos de género en la Ley General de Población y su marco regulador en México. Fundó la Cátedra Universitaria ACNUR Sergio Vieira de Mello en materia de derechos humanos para 22 universidades públicas de América del Sur. En 2019 fue reconocida por la Comisión Nacional de los Derechos Humanos. Es miembro del equipo de capacitadores de FUPAREDEH y exdirectora ejecutiva del Instituto Nacional de Migración (INM RD), 2013-2020. 